\title{
Dénombrement des Champignons kératinophiles d'une population de Cynocéphales (Papio papio)
}

\author{
Par F. MARIAT et Gladys TAPIA ${ }^{1}$
}

L'existence dans la nature, à l'état saprophytique, de champignons kératinophiles souvent potentiellement pathogènes, constitue un fait d'un grand intérêt épidémiologique. Depuis la découverte de Microsporum gypseum dans le sol par M.-A. Gordon (1953), puis par L. Ajello (1953), on s'est efforcé de déterminer les sources éventuelles d'infection, par la recherche systématique dans le sol ou sur les animaux des champignons kératinophiles ou dermatophytes. L'emploi de méthodes nouvelles (Vanbreuseghem, 1952; Georg et al., 1954), rendant aisé leur isolement à partir de la nature, a permis de montrer la relative fréquence de ces champignons dans les substrats naturels.

On trouve dans la littérature de nombreuses références se rapportant à la recherche systématique de champignons kératinophiles chez l'animal: de multiples observations effectuées sur des espèces variées ont été publiées. Les singes qui peuvent être éventuellement atteints de dermatophyties diverses n'avaient cependant jamais fait l'objet de telles enquêtes. Il nous a donc paru intéressant de mettre à profit une opportunité qui nous était offerte d'étudier une population de cynocéphales africains. Le travail développé ci-dessous a fait l'objet d'une courte note préliminaire (Tapia et Mariat, 1965).

\section{Matériel et méthodes}

Au cours des mois de novembre 1964 à mars 1965 nous avons examiné 115 cynocéphales (Papio papio) dont 62,6\% d'animaux mâles. Les singes originaires de Guinée, et du Kenya, vivaient à Paris depuis des temps variables généralement courts (de deux à huit semaines) dans une singerie bien aménagée. Beaucoup d'animaux, avant d'arriveı à Paris, avaient séjourné à Londres, en transit, pendant un bref délai.

Un groupe de 105 singes a fait l'objet d'une étude détaillée : examen clinique sous anesthésie (en lumière normale et en lumière de Wood), puis prélèvement de matériel en huit endroits différents. Dix autres animaux n'ont subi que deux prélèvements. Ces derniers singes ont été, par la suite, observés pendant plusieurs semaines et utilisés aux inoculations expérimentales.

Les prélèvements, au niveau des poils, sur les animaux du premier groupe ont été effectués par l'emploi de deux techniques. La première est une méthode originale inspirée des techniques proposées par Gentles (1956) et par Mackenzie (1961) : à l'aide d'un morceau de tapis de laine (moquette) d'environ $6 \times 6 \mathrm{~cm}$, préalablement lavé et stérilisé, on brosse énergiquement en tous sens la région choisie du corps, (tête, dos, ventre).

(1) Nous remercions également MM. L. Chaumont et E. Le Brocher de nous avoir aidés à manipuler des animaux souvent peu compréhensifs. 
Le morceau de moquette ainsi souillé permet d'ensemencer successivement une plaque de milieu gélosé et glucosé de Sabouraud, puis une plaque de milieu maltosé. Les milieux contiennent $0,5 \%$ de chloramphénicol et $0.5 \%$ de cycloheximide ( Actidione »). L'ensemencement est pratiqué en appliquant à plusieurs reprises les poils du tapis sur la surface de la plaque de milieu. Les avantages de cette méthode sont dus à la nature même du tapis qui réalise une brosse de laine à poils serrés permettant un prélèvement efficace, mais aussi à la facilité d'emploi et de réemploi en condition d'asepsie. Les morceaux de moquette ont été également utilisés avec succès pour effectuer des prélèvements variés (sols, murs, plafonds, etc.), ou même comme substrat de culture.

La seconde technique, classiquement employée, consiste à mettre en culture sur de la terre stérile ou sur une plaque de milieu glucosé de Sabouraud aux antibiotiques, des poils coupés sur la tête des animaux.

Outre les poils, d'autres prélèvements ont été effectués : de la poudre des ongles de la main obtenue à l'aide d'une lime émeri flambée, des squames interdigitales des mains et des squames de la région inguino-crurale obtenues par râclage au bistouri. Tous ces produits sont mis en culture en tubes de milieu de Sabouraud additionné d'antibiotiques.

Parallèlement à ces prélèvements et après avoir noté la présence fréquente de Candida albicans dans les cultures, des selles ont été prélevées par écouvillonnage rectal : L'écouvillon permet d'ensemencer des milieux de Sabouraud contenant des antibiotiques.

Pour les animaux du second groupe on procède seu'ement à un prélèvement de poils coupés sur la tête et mis en culture sur terre et à un prélèvement sur le dos par la méthode du tapis.

Toutes les cultures sont incubées à $25^{\circ}$. Les boîtes de Petri sont fermées par un ruban adhésif et des plaques témoins non ensemencées sont également disposées dans l'étuve. L'identification des champignons est faite d'après la morphologie macro- et microscopique en utilisant les méthodes usuelles.

\section{Résultats}

EXAmen clinique: Sur les 115 animaux examinés, 3 seulement $(2,6 \%)$ présentaient des lésions teigneuses caractéristiques, les autres en paraissaient exempts. Les dermatophytes suivants ont été isolés des lésions d'alopécie diffuse, desquamatives et non inflammatoires: Microsporum cookei (singe $\mathrm{n}^{\circ}$ 53, Souche I.P. 913), M. gypseum (singe $\mathrm{n}^{\circ} 982$ ) et un Trichophyton endotriche proche de T. rubrum (singe $\mathrm{n}^{\circ} 978$ ). La souche de $M$. cookei spontanément pathogène pour le singe devait également se révéler par la suite expérimentalement pathogène. La souche de Trichophyton endotriche et la souche de $M$. gypseum se sont également montrées pathogènes expérimentalement.

De nombreux singes (environ $80 \%$ ) présentaient des nodules blanchâtres à fluorescence jaune-vert, engainant certains poils sur 1 ou $2 \mathrm{~mm}$ de longueur. Ces nodules sont d'origine bactérienne. Sur aucun animal nous n'avons noté la présence de nodules de piedra tels que ceux décrits par Kaplan (1959 $a$ et $b)$.

Champignons kératinophiles isolés : Alors que seulement trois singes sur 115 présentaient des lésions teigneuses provoquées par les dermatophytes mentionnés plus 
haut, le nombre des animaux sains porteurs de champignons kératinophiles a été considérable. Sur 112 singes apparemment sains, 61 soit 54,4\% étaient infestés. A partir de 57 d'entre-eux, 105 souches de Trichophyton mentagrophytes ont été isolées. On a également isolé $M$. cookei dans 3 cas et une souche de Keratinomyces ajelloi dans 1 cas. Le tableau I donne le détail de ces isolements. En dehors des champignons mentionnés, de nombreux saprophytes (Aspergillus, Penicillium, Cladosporium, Cephalosporium, Allescheria, etc..., etc...), ont été obtenus en culture, identifiés sommairement et rejetés. Nous n'avons conservé que les seuls champignons pour lesquels on hésite à utiliser l'épithète kératinophile ou dermatophyte (Rioux et al., $1965 \mathrm{~b}$ ).

Tableau I

SiNGES PORTEURS DE CHAMPIGNONS KÉRATINOPHILES (population totale : 115 Cynocéphales)

\begin{tabular}{|c|c|c|c|c|c|}
\hline \multirow{3}{*}{$\begin{array}{c}\text { Champignons } \\
\text { isolés }\end{array}$} & \multicolumn{5}{|c|}{ Nombre d'animaux porteurs } \\
\hline & \multirow{2}{*}{$\begin{array}{l}\text { Teigneux } \\
\text { (3) }\end{array}$} & \multicolumn{2}{|c|}{$\begin{array}{c}\text { Apparemment sains } \\
\text { (112) }\end{array}$} & \multicolumn{2}{|c|}{ Nbre total (115) } \\
\hline & & & de 112 & & de 115 \\
\hline \multirow{7}{*}{$\begin{array}{l}\text { T. mentagrophytes var. } \\
\text { granulosum } \ldots \ldots \ldots \ldots \ldots \ldots \\
\text { T.m. var asteroides } \ldots \ldots \ldots \\
\text { M. cookei } \ldots \ldots \ldots \ldots \ldots \ldots \\
\text { M. gypseum } \ldots \ldots \ldots \ldots \ldots \ldots \\
\text { K. ajelloi } \ldots \ldots \ldots \ldots \ldots \ldots \\
\text { T. rubrum } \ldots \ldots \ldots \ldots \ldots \ldots\end{array}$} & - & 51 & 45,5 & 51 & 44,3 \\
\hline & 一 & 6 & 5,3 & 6 & 5,2 \\
\hline & 1 & 3 & 2,7 & 4 & 3,5 \\
\hline & 1 & - & - & 1 & 0,9 \\
\hline & - & 1 & 0,9 & 1 & 0,9 \\
\hline & 1 & - & - & 1 & 0,9 \\
\hline & 3 & 61 & 54,5 & 64 & 55,6 \\
\hline
\end{tabular}

Le tableau II montre la répartition des isolements des 103 souches de $T$. mentagrophytes effectués à partir des 55 animaux positifs du premier groupe de 105 singes. On remarque que, chez un même animal, un ou plusieurs prélèvements peuvent être positifs : si 30 singes ne donnent qu'un seul prélèvement positif, d'autres en permettent jusqu'à cinq et même sept. Ce tableau montre également qu'à partir de la tête, l'isolement de $T$. mentagrophytes n'est parfois obtenu qu'à l'aide d'une seule des deux méthodes employées : la mise en culture d'une mèche de poils coupés se montre ici plus favorable à l'isolement du champignon.

Les souches de $T$. mentagrophytes isolées sont riches en éléments caractéristiques : macroconidies cylindriques, pluriloculaires, parfois ramifiées ou même trifurquées, microconidies sphériques et vrilles. Cultivées sur crin de cheval ou poils de singe sur 


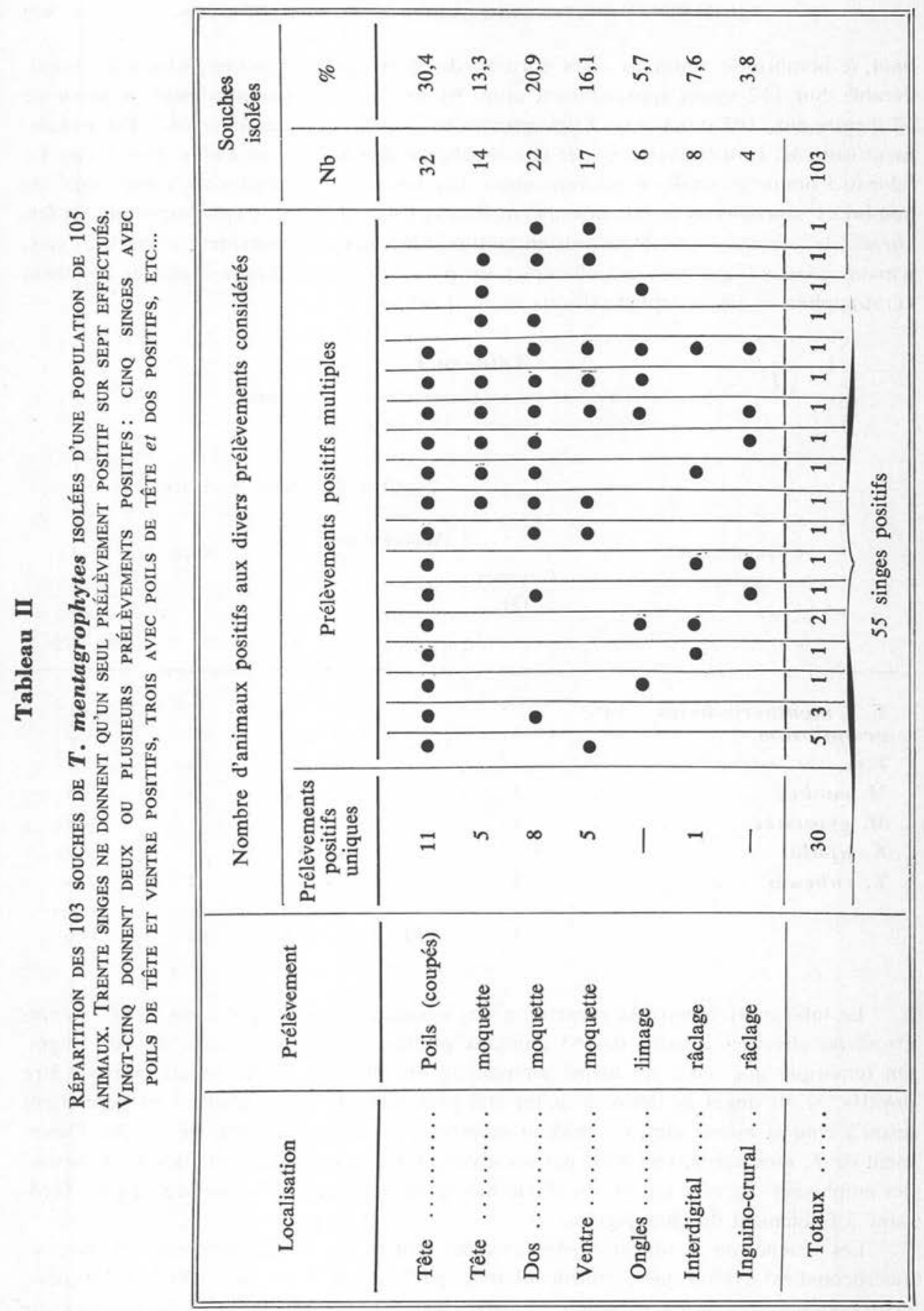


terre stérile, la majorité d'entre elles forment des « fruits conidiens » (Matruchot et Dassonville, 1900): appareils globuleux blanchâtres limités par une paroi lâche d'où émergent des filaments ressemblant aux hyphes péridiaux du genre Arthroderma et contenant en leur centre une multitude de microconidies. En aucun cas, après de multiples essais de croisement de souches, la formation de cleistothèces fertiles n'a été obtenue. Le pléomorphisme atteint rarement ces souches florides, à développement rapide qui sont très comparables aux $T$. mentagrophytes isolés du sol par Rioux et al. (1965 $a$ ).

Toutes les souches inoculées au cobaye se sont révélées fortement pathogènes.

Sur la base de la morphologie, nous avons réparti les souches isolées entre les variétés granulosum et asteroides telles que les concevait Sabouraud (1910). Toutes cependant contenaient d'abondantes macroconidies. Les souches rangées dans l'une ou l'autre variété appartiennent à la forme granulaire de $T$. mentagrophytes définie par Georg (1954).

Les souches de $M$. gypseum, de $K$. ajelloi de même que les quatre $M$. cookei sont conformes aux descriptions classiques. Rappelons cependant que l'un de ces derniers s'est montré spontanément et expérimentalement pathogène et qu'il présente quelques particularités morphologiques secondaires.

Le Trichophyton endotriche qu'actuellement nous rapprochons de $T$. rubrum, diffère de cette dernière espèce par une formation réduite de pigment rouge, limitée d'ailleurs au bord externe de la colonie. Le pigment est le plus souvent de teinte jaune à jaune-orange : dans les premières rétrocultures, les microconidies étaient abondantes et les macroconidies caractéristiques, fréquentes. Elles le sont encore dans certaines cultures.

Les champignons kératinophiles rencontrés sur la fourrure des singes se retrouvent également dans les locaux où sont gardés les animaux. Des boîtes de Petri contenant de la terre et des crins de cheval stériles, ouvertes pendant une semaine en 18 endroits différents des cinq salles de la singerie, ont permis d'isoler cinq champignons kératinophiles : trois $T$. mentagrophytes, un $M$. gypseum et un Trichophyton sp. Les spores qui ont ainsi contaminé les boîtes ont été véhiculées par l'air. Neuf autres prélèvements effectués en frottant une surface de $20 \times 20 \mathrm{~cm}$ de plafond ou de paroi nous ont permis d'isoler une seule souche de $T$. mentagrophytes dont les spores adhéraient au support. Un prélèvement pratiqué sur les gants servant à manipuler les animaux s'est montré négatif.

Isolement de Candida albicans. Sur les 115 animaux, 54, soit 46,9 \%, étaient infestés par $C$. albicans qu'on a isolé de différentes localisations. Les animaux se contaminant sans doute secondairement par salissures d'excréments, nous avons pour 81 d'entre eux effectué des cultures de selles prélevées par écouvillonnage rectal : 19 prélèvements ont donné des colonies de $C$. albicans. Ainsi plus de $23 \%$ des animaux hébergent l'espèce $C$. albicans dans leur tube digestif. Nous n'avons jamais isolé d'autres espèces de Candida. L'actidione des milieux d'isolement peut, il est vrai, éliminer certaines d'entre elles. 


\section{Discussion}

Cette étude fait ressortir que de nombreux cynocéphales apparemment exempts de dermatophyties sont porteurs de champignons kératinophiles potentiellement pathogènes. Près de $50 \%$ des individus de la population étudiée hébergent des souches de $T$. mentagrophytes capables de provoquer de larges lésions inflammatoires expérimentales chez le cobaye et chez le singe. Il est étonnant de constater qu'un cynocéphale portant $T$. mentagrophytes sur plusieurs régions de son corps et par ailleurs constamment exposé aux blessures les plus diverses, en particulier aux morsures de ses congénères, ne présente pas de lésion teigneuse évolutive due à ce champignon. Une immunité, acquise au contact permanent du champignon, pourrait expliquer ce fait, mais il ne semble pas que cela soit. Des essais ont été effectués sur des animaux porteurs et non porteurs de $T$. mentagrophytes: les uns comme les autres ont présenté une lésion teigneuse très active après inoculation expérimentale de T. mentagrophytes; ils ont réagit de semblable manière. Dans ces essais les singes trouvés porteurs du champignon ont été inoculés avec leur souche homologue.

On ne peut cependant incriminer une résistance du singe aux teignes spontanées. On sait en effet que celles-ci ne sont pas exceptionnelles et des travaux récents le rappellent: Klokke et de Vries (1962) avec Microsporum obesum chez le chimpanzé, Seeliger, Bisping et Brandt (1963) avec $M$. canis chez le gibbon, Koch et Jänisch (1964) avec $M$. gypseum chez le rhesus, Franquet et Lectard (1965) avec $M$. gypseum chez le mandrill, et enfin Phyllis Stockdale et al. (1965) avec Trichophyton simii chez le rhesus. Ces publications mentionnent des observations plus anciennes, notamment celles de Pynoy (1912) et d'Emmons (1940). Nous avons nous-même constaté au cours de cette enquête que trois singes $(2,6 \%$ seulement il est vrai) présentaient des lésions spontanées.

Les singes étudiés vivaient en captivité ; les conditions inhérentes à l'habitat en cages où les animaux sont conservés par lots de 2 à 5 individus, ainsi que tous les facteurs découlant de la capture et de la captivité expliquent peut-être le haut pourcentage d'animaux infestés. Nous avons par exemple montré l'existence du champignon dans l'air et sur les parois des salles où sont gardés les animaux. Notons cependant que, à l'état libre, le cynocéphale est un animal coureur, vivant le plus souvent au contact du sol, et qu'il est fort probable qu'il s'infecte, à l'origine, à partir de celui-ci. Plus qu'un réservoir, représenté en réalité par le sol lui-même, le cynocéphale, simple support du champignon, serait plus exactement un véhicule particulièrement actif du micro-organisme. L'infestation de l'animal se ferait à partir du sol dans des conditions semblables à celles qu'ont proposées Rioux et al. (1965 a). Nous nous préoccupons actuellement de connaître le pourcentage d'infestation des cynocéphales vivant à l'état libre sur les savanes africaines.

Notre enquête était terminée quand est paru l'intéressant mémoire de P. Stockdale et al. (1965) sur Trichophyton simii. Nous n'avons donc étudié, comparativement à cette espèce, que quelques-unes de nos souches de $T$. mentagrophytes. Il ne nous est pas possible, du fait de la grande similitude entre les deux entités, d'affirmer qu'aucune 
des 105 souches de $T$. mentagrophytes isolées du cynocéphale n'appartient à l'espèce $T$. simii. La morphologie de l'ensemble de nos souches, mais aussi les essais infructueux de produire des cleistothèces fertiles, nous incitent cependant à penser qu'au moins la majorité des souches appartiennent plutôt à l'espèce $T$. mentagrophytes qu'à l'espèce T. simii. Quelques souches récentes ont été étudiées par P. Stockdale et D. W. R. Mackenzie (1966). Ce dernier a obtenu des cleistothèces fertiles en croisant notre souche $F 12$ et la souche IMI 101694 d'Arthroderma (T.) simii * + ».

L'isolement d'une souche de $M$. cookei spontanément pathogène pour le singe et expérimentalement pathogène pour cet animal et, à un degré moindre, pour le cobaye est un fait qui doit être souligné. $M$. cookei est en effet une espèce géophile jusqu'alors considérée comme non pathogène. Cette souche $[M$. cookei I.P. $913=$ Nanizzia cajetana $(+)$ ] fait l'objet d'une note publiée par ailleurs (Mariat et Tapia 1966).

\section{Résumé}

On étudie la fréquence et la répartition des champignons kératinophiles potentiellement pathogènes sur la peau et les annexes cutanées de 115 Cynocéphales africains (Papio papio) vivant en captivité. Chaque animal a été examiné en lumière de Wood et en lumière normale et il a fait l'objet de prélèvements systématiques. Les prélèvements au niveau des poils ont été effectués notamment à l'aide d'une méthode originale (méthode du tapis). Les divers prélèvements : poils de diverses régions, ongles, squames interdigitales et inguino crurales, ont permis d'obtenir en culture un total de 112 souches de champignons kératinophiles isolés à partir de 64 individus d'une population de 115 singes. Trois seulement des animaux observés $(2,6 \%)$ présentaient des lésions teigneuses caractérisées, provoquées par Microsporum cookei, M. gypseum et par un Trichophyton proche de $T$. rubrum. Des animaux apparemment sains on a isolé 105 souches de $T$. mentagrophytes (six $T . m$. var. asteroides et $99 T . m$. var. granulosum) à partir de 57 singes, trois souches de $M$. cookei à partir de trois singes et une souche de K. ajelloi.

Les souches de $T$. mentagrophytes sont riches en organes caractéristiques : microet macroconidies, vrilles; cultivées sur sol, seules ou en mélange, elles produisent d'abondants « fruits conidiens ».

Des prélèvements effectués dans l'animalerie ont permis d'isoler trois souches de $T$. mentagrophytes, une de $M$. gypseum et une de Trichophyton sp.

Par ailleurs Candida albicans a été isolée des selles de $23,4 \%$ des singes.

Dans la discussion on envisage le rôle des cynocéphales comme véhicules des champignons kératinophiles dont le sol constitue un réservoir. On considère comme probable, mais cela reste à démontrer, que les conditions dues à la captivité augmentent la fréquence des champignons kératinophiles. On s'étonne que des animaux si fortement infestés par $T$. mentagrophytes et exposés à de fréquents traumatismes ne présentent pas de teignes évolutives dues à ce champignon.

\section{Bibliographie}

AJELlo L., 1953. - The dermatophyte Microsporum gypseum, as a saprophyte and parasite. Jour. Invest. Derm., 21, 157-171. 
Emmons C. W., 1940. - Trichophyton mentagrophytes (Pinoyella simii) isolated from dermatophytosis in the monkey. Mycopathologia et Mycol. applic., 2, 317-319.

FRANQUet R. et Lectard P., 1965. - Dermatophytie chez un mandrill de Brazzaville. Bull. Soc. Fr. Mycol. Méd., $\mathrm{n}^{\circ}$ 9, p. 9.

Gentles J. C., 1956. - The isolation of dermatophytes from the floors of communal bathing places. J. Clin. Path., 9, 374-377.

Georg L. K., 1954. - The relationship between the downy and granular forms of Trichophyton mentagrophytes. J. Invest. Dermat., 23, 123-141.

Georg L. K., Ajello L. and Papageorge C., 1954. - Use of cycloheximide in the selective isolation of fungi pathogenic to man. Jour. Lab. Clin. Med., 44, 422-423.

Gordon M. A., 1953. - The occurrence of the dermatophyte Microsporum gypseum as a saprophyte in soil. Jour. Invest. Derm., 20, 201-206.

Kaplan W., 1959 a. - Piedra in lower animals. A case report of white piedra in a monkey and a review of the literature. J. Am. Vet. Med. Ass., 134, 113-117.

KaPLAN W., 1959 b. - The occurrence of black piedra in primate pelts. Trop. Georg. Med., $11,115-126$.

KlokKe A. H. and de Vries G. A., 1962. - Tinea capitis in chimpanzees caused by Microsporum canis Bodin 1902 resembling $M$. obesum Conant 1937. Sabouraudia, 2, 268-270.

КосH H. A. und JANISCH W., 1964. - Eine Mikrosporum gypseum Enzootie bei Rhesusaften (Macacus rhesus). Mykosen, 7, 86-89.

MackenziE D. W. R., 1961. - The extra human occurrence of Trichophyton tonsurans var. sulfureum in a residential school. Sabouraudia, 1, 58-64.

MACKENZIE D. W. R., 1966. - Communication personnelle.

MARIAT F. et TAPIA G., 1966. - Observations sur une souche de Microsporum cookei parasite du cynocéphale (Papio papio). Sabouraudia, 5, 43-45.

Matruchot L. et Dassonville C., 1900. - Sur une forme de reproduction d'ordre élevé chez les Tricophyton. Bull. Soc. Mycol. Fr., 16, 201-208.

PinOY E., 1912. - Sur une teigne cutanée du singe. C.R. Soc. Biol., 72, 59.

RIouX J.-A., JARRY D.-T., JARRY D.-M. et BouReLly C., 1965 a. - Isolement de Trichophyton mentagrophytes des sols du Sud de la France. Sabouraudia, 4, 11-16.

RIOUX J.-A., JARRY D.-M. et JARRY D.-T., 1965 b. - Ctenomyces, Arthroderma ou Trichophyton. Fin d'une controverse et nouvelle acception du terme de dermatophyte. Bull. Soc. Fr. Myc. méd., n 10, p. 7.

Sabouraud R., 1910. - Les Teignes, Masson édit., Paris.

SEeliger H.P. R., Bisping W. und Brandt N.P., 1963. - Ueber eine Mikrosporum Enzootie bei kappen-Gibbons verursacht durch eine Variante von Microsporum canis. Mykosen, 6, 61-68.

StOCKDAle P. M., 1966. - Communication personnelle.

Stockdale P. M., Mackenzie D. W. R. and Austwick P. K. C., 1965. - Arthroderma simii sp. nov., the perfect state of Trichophyton simii (Pinoy) comb. nov. Sabouraudia, 4 112-123.

TAPIA G. et Mariat F., 1965. - Champignons kératinophiles des singes africains. Bull. Soc. Fr. Myc. méd., n ${ }^{\circ}$ 9, p. 5.

VANBREUSEGHEM R., 1952. - Technique biologique pour l'isolement des dermatophytes du sol. Ann. Soc. Belge Med. Trop., 32, 173-178.

(Institut Pasteur, Paris, Service de Mycologie, et Institut d'Hygiène, Caracas) 